\title{
A Robot Supervision Architecture for Safe and Efficient Space Exploration and Operation
}

\author{
Ehud Halberstam, Luis Navarro-Serment, Ronald Conescu, Sandra Mau, \\ Gregg Podnar, Alan D. Guisewite, H. Benjamin Brown, Alberto Elfes*, \\ John M. Dolan, Marcel Bergerman
}

Robotics Institute, Carnegie Mellon University, 5000 Forbes Avenue, Pittsburgh, PA, 15213-3890; Tel.: +1-412-268-7988; email: jmd@ @cs.cmu.edu

"Jet Propulsion Laboratory, 4800 Oak Grove Drive, Pasadena, CA, 91109-8099; Tel.: 1-818-393-9074; email: elfes@jpl.nasa.gov

\begin{abstract}
Current NASA plans envision human beings returning to the Moon in 2018 and, once there, establishing a permanent outpost from which we may initiate a long-term effort to visit other planetary bodies in the Solar System. This will be a bold, risky, and costly journey, comparable to the Great Navigations of the fifteenth and sixteenth centuries. Therefore, it is important that all possible actions be taken to maximize the astronauts' safety and productivity. This can be achieved by deploying fleets of autonomous robots for mineral prospecting and mining, habitat construction, fuel production, inspection and maintenance, etc.; and by providing the humans with the capability to telesupervise the robots' operation and to teleoperate them whenever necessary or appropriate, all from a safe, "shirtsleeve" environment.

This paper describes the authors' work in progress on the development of a Robot Supervision Architecture (RSA) for safe and efficient space exploration and operation. By combining the humans' advanced reasoning capabilities with the robots' suitability for harsh space environments, we will demonstrate significant productivity gains while reducing the amount of weight that must be lifted from Earth - and, therefore, cost.

Our first instantiation of the RSA is a wide-area mineral prospecting task, where a fleet of robots survey a pre-determined area autonomously, sampling for minerals of interest. When the robots require assistance - e.g., when they encounter navigation problems, reach a prospecting site, or find a potentially interesting rock formation they signal a human telesupervisor at base, who intervenes via a high-fidelity geometrically-correct stereoscopic telepresence system (Figure 1a). In addition to prospecting, the RSA applies to a variety of other tasks, both on the surface: mining, transporting, and construction - and on-orbit: construction, inspection, and repair of large space structures and satellites (Figure 1b).
\end{abstract}




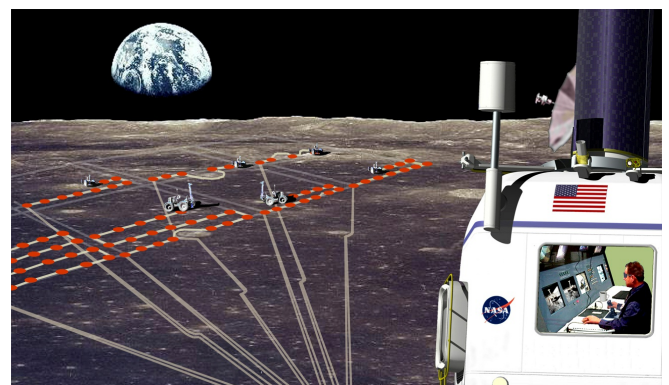

(a)

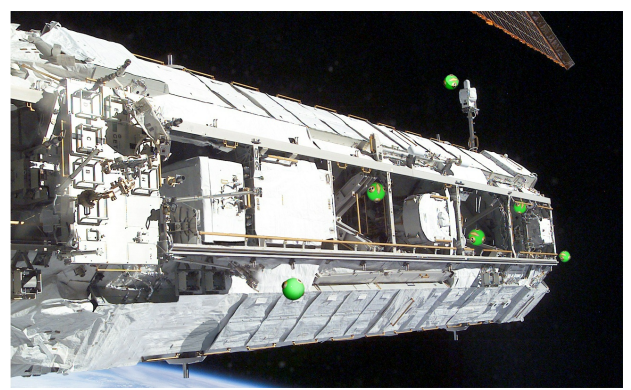

(b)

Figure 1. (a) Artist's concept of telesupervised wide-area robotic prospecting on the Moon. (b) Concept of telesupervised space station truss inspection.

This paper is structured as follows: In the following section we present related work and emphasize the contribution we bring to the state-of-the-art. Next, we describe the robot supervision architecture, which is the overarching paradigm under which all other modules function. We then turn our attention to the main focus of the paper, our current system implementation and results, where three rovers at Carnegie Mellon University (CMU), NASA Ames Research Center (ARC) and NASA Jet Propulsion Laboratory (JPL) visit targets simulating regions of interest for prospecting, both in autonomous and teleoperated modes. The paper closes with conclusions and plans for future work.

\section{Literature Review}

The human-robot interaction literature has been growing steadily in the past few years. While part of the research community is addressing problems related to robot sociability [5], we and others are investigating using robots as tools that increase human safety and productivity in hazardous environments. The current state of the art in robotic space exploration is the Mars Exploration Rover (MER) Mission, with its two rovers, Spirit and Opportunity, having logged more than $10 \mathrm{~km}$ [7] combined. Although tremendously successful, the MER rovers each require a team of 20 or more professionals to plan and monitor their progress. Researchers who have been promoting an alternative scenario, i.e. one human supervising a fleet of robots, include Fong et al. [3], Heger et al. [4], Nourbakhsh et al. [8], and Sierhuis et al. [10]. For the sake of brevity, we indicate here that our work differs from these either in underlying assumptions, algorithms, applications targeted, or technology readiness levels sought [6]. A more detailed review is provided in [1].

\section{The Robot Supervision Architecture}

The Robot Supervision Architecture (RSA) is an evolution of ATLAS, a multilayered replicated architecture developed by Elfes [2]. The multilayered property comes from the robot system control being performed at multiple levels of resolution and abstraction, at different control rates; and the replicated property from the fact that the perception, decision-making and actuation activities occur at each level of the architecture.

Figure 2a presents the high-level block diagram of the RSA. At the telesupervision workstation, four main processes coexist: Task Planning and Monitoring, Robot Telemonitoring, Telepresence and Teleoperation, and Robot Fleet Coordination. At 
each robot, Robot Controller and Hazard and Assistance Detection (HAD) processes are responsible for task execution. Figure $2 \mathrm{~b}$ presents our concept of the telesupervisor workstation. At the center, a high-bandwidth stereoscopic image allows for telepresence and teleoperation. To the left are the Task Planning and Monitoring tools, and to the right the Robot Telemonitoring ones. For more details on the RSA components and processes we refer the reader to [1], [9]; their current implementation at the workstation side is described in the following section.

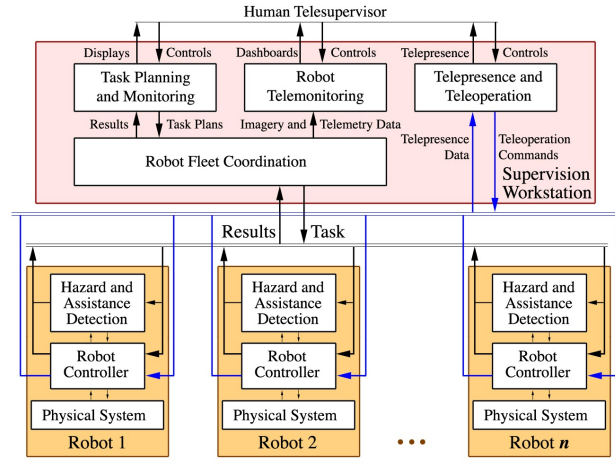

(a)

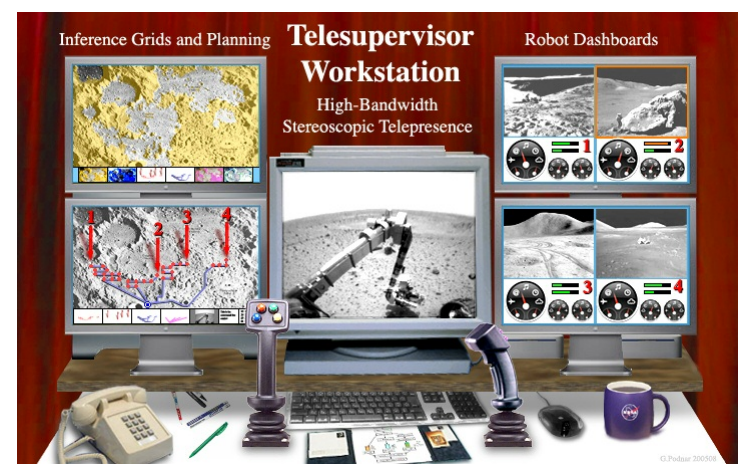

(b)

Figure 2. Robot Supervision Architecture. (a) High-level block diagram. (b) Concept of the telesupervisor workstation.

\section{Implementation}

Our first instantiation of the RSA is focused on wide-area prospecting of minerals and water. Our goal is to autonomously search for in situ resources in a terrestrial field analogue to the Moon or Mars, with the human telesupervisor being able to assist any robot in the fleet whenever necessary. California and Arizona provide convenient and accessible field-test sites for characterizing robotic/human performance and system integration. Our team's geologist has established several rover study sites in the Mojave near Silver Dry Lake and Edwards Air Force Base for the highly successful Mars Exploration Rover test program.

\section{$\underline{\text { Robot Fleet }}$}

Our robot fleet is currently composed of four vehicles: two K10s, at NASA ARC and CMU and two Sample Return Rovers (SRR) at JPL (Figure 3).

The K10s are four-wheel vehicles designed and built by NASA ARC. It is composed of the following systems: mechanical, power, communications, and electronic motor control. The mechanical system includes the main frame, protective panels, a rockerbeam suspension system, and four independent wheels. The rocker-beam arrangement increases the rover's ability to negotiate obstacles, and offers a simpler and cheaper alternative to the rocker-bogie suspension system used in other planetary rovers, such as NASA JPL's K9 or FIDO. In this system, an angular displacement produced in one rocker-beam is reflected in the opposite direction in the other beam through a set of pushrods and a pivot pin.

The SRRs are small four-wheel rovers that employ four-wheel independent drive and steering. Both are capable of speeds up to $10 \mathrm{~cm} / \mathrm{s}$. Each rover is equipped with a 
3 degrees-of-freedom "micro-arm" with an actuated gripping end-effector. A forward-looking stereo camera pair $\left(120^{\circ}\right.$ field of view) is used for obstacle detection.

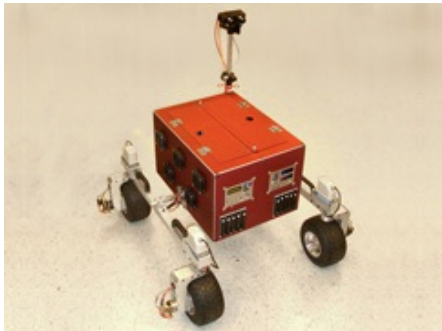

(a)

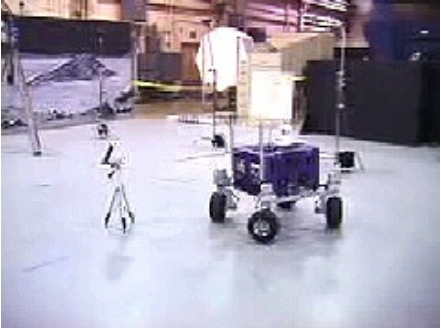

(b)

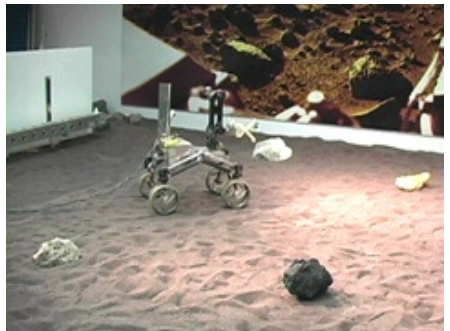

(c)

Figure 3. (a), (b) The K10s at CMU and NASA ARC. (c) The SRR at NASA JPL.

\section{Task Planning and Monitoring}

The task planning and monitoring tool allows us to define a set of waypoints for the robots to traverse and sample points where prospecting takes place. The current version is shown in Figure 4. It contains ten panels that allow for the following functionalities (please refer to the numbers in Figure 4):

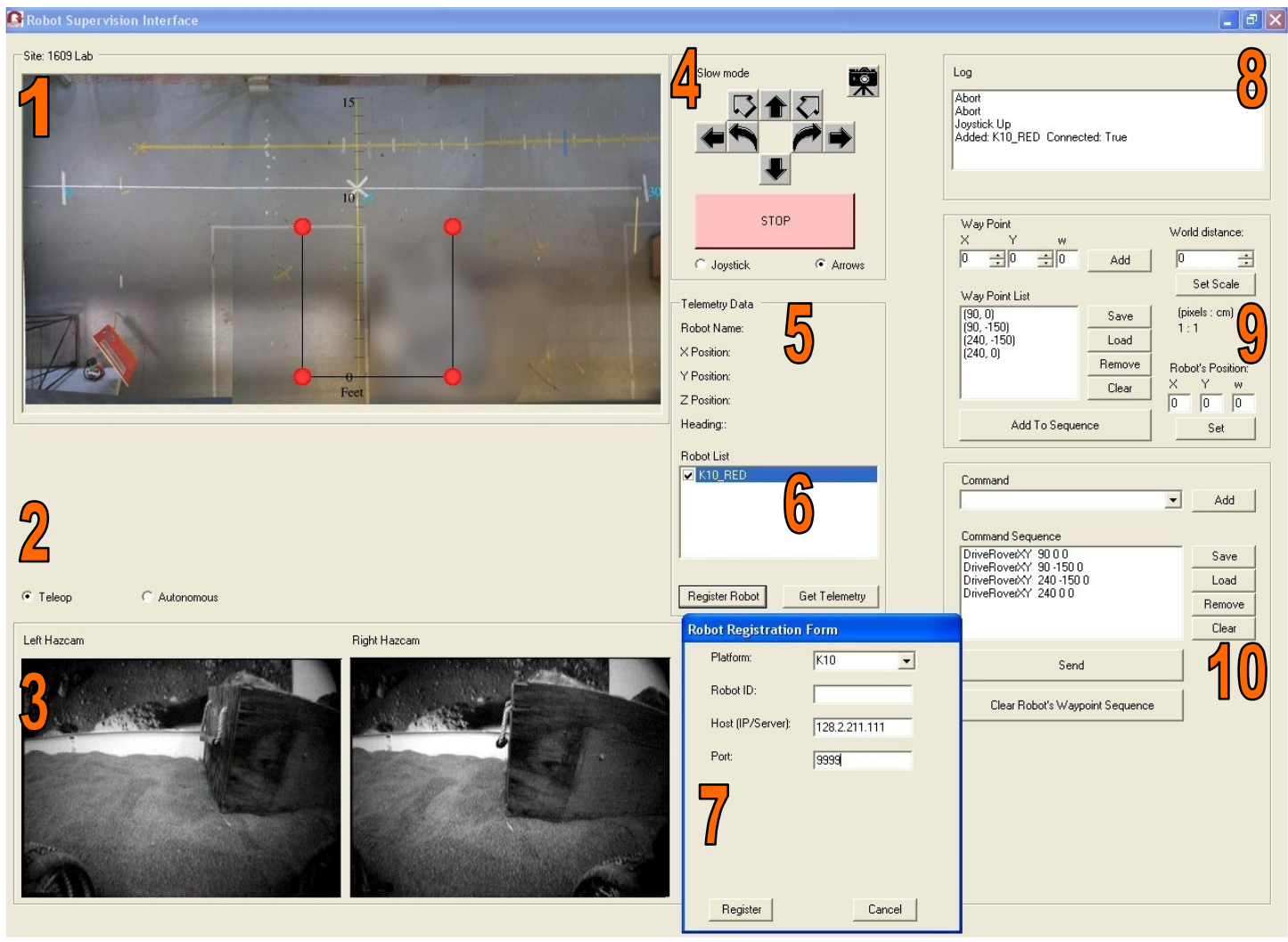

Figure 4. RSA task planning and monitoring tool.

1. The Site Panel displays an overhead image of the area to be covered. While testing the system indoors and on Earth we use pictures and satellite-based imagery. When the system is deployed in an actual extraterrestrial environment, satellite-based imagery with the highest resolution possible will be used. The site panel is used to define the set of waypoints that a robot must traverse. These are defined by clicking 
the mouse on the desired destination in the site image, and can be edited or deleted before being assigned to the robot. This panel will also show the actual path traversed overlaid on the path planned, for mission analysis and assistance detection purposes.

2. The Teleoperation/Autonomous switch is used to toggle between operation modes any time during a robot's task execution.

3. The Robot View Display panel displays imagery received from a selected robot at a fixed update rate.

4. The Direct Control panel allows the user to directly teleoperate a robot using the mouse, by clicking on the appropriate arrows.

5. The Telemetry Data panel displays the robot's telemetry data, indicating its current state and position. Telemetry data are updated at a fixed rate.

6. The Robot List panel lists all the robots in the fleet recognized by the task planning and monitoring tool, and allows the user to select which robot to interact with.

7. The Robot Registration Form is used to add robots to the list of supervised robots recognized by the tool.

8. The Log panel displays text messages of events that are of interest to the user.

9. The Waypoint panel is used to display and edit waypoints. Pressing the 'Add To Sequence' button will translate the waypoint list to the path traversal command sequence that is ultimately sent to the robot. Designated paths can be saved and reloaded as needed. In addition, this panel is used to determine map scale and coordinate system origin, and to reset a robot's internal coordinate system.

10. The Command Sequence panel is used to display and edit control command sequences. Robot control commands include the set of commands that are supported by each robot in the fleet. Command sequences can be saved and reloaded as needed.

\section{Telepresence and Teleoperation}

At the center of the telesupervisor workstation lies the telepresence and teleoperation console. Figure 5a presents its current implementation, based on a custom-designed geometrically-correct stereoscopic camera (Figure 5b; see also the left image on Figure 3 for the camera mounted on the K10 robot), a StereoGraphics Corp. Z-screen, and a standard joystick. In our camera, the image sensors are coplanar and the optical axes are parallel. To achieve coincident centers of view, instead of converging the optical axes (the traditional solution in the computer vision area) we are able to independently shift the center of view of each camera by shifting each image sensor while keeping them coplanar. The result is an undistorted, high-fidelity view from the robot's cameras as if the telesupervisor was sitting on or in the robot. We use a highbandwidth commercial video link to transmit imagery from the cameras to the workstation, with a low-bandwidth version to allow for graceful fallback.

\section{Robot Telemonitoring}

The Robot Telemonitoring process comprises two software modules: the Robot Poller and the Dashboard. Several times each second, the Robot Poller gathers information from various sensors on each robot, and stores that information in a time-stamped XML file on the telesupervisor workstation. From there, a standard Web server makes the XML file available to any client on our local network. The XML file contains the 
robot's status information formatted in such a way that both humans and computers can easily interpret it.

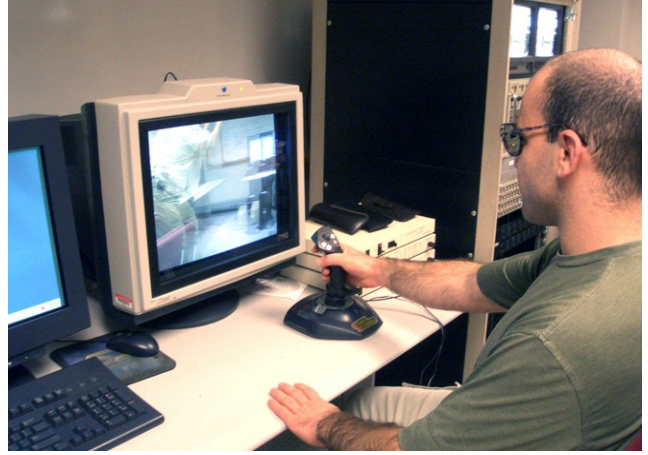

(a)

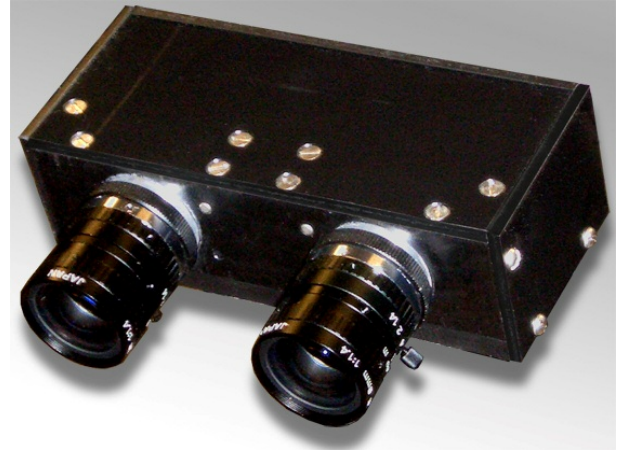

(b)

Figure 5. (a) Telepresence workstation. (b) Stereoscopic camera.

Likewise, several times each second, the Dashboard requests the XML file and presents each sensor's data appropriately in a graphical format reminiscent of standard aircraft instrument panels. For example, Figure 6 presents the Dashboard as CMU's K10-red robot (robot ID 001 in the upper left corner) navigates through a simulated course. Note that the roll angle in the artificial horizon is $10^{\circ}$, which is above the alert threshold of the HAD system. Therefore, the light indicator to the right of the roll reading appears yellow. In the same way, the fuel indicator is in emergency mode because of the low battery level, and announces that by setting the corresponding light to red. The GPS sensor, on the other hand, indicates that data from at least six satellites can be read, a normal operating condition not signaled by the GPS light indicator. A compass is also available to present the robot's heading. Because one of the sensors invokes emergency mode, the border of the Dashboard turns red to alert the human telesupervisor to the imminent problem.

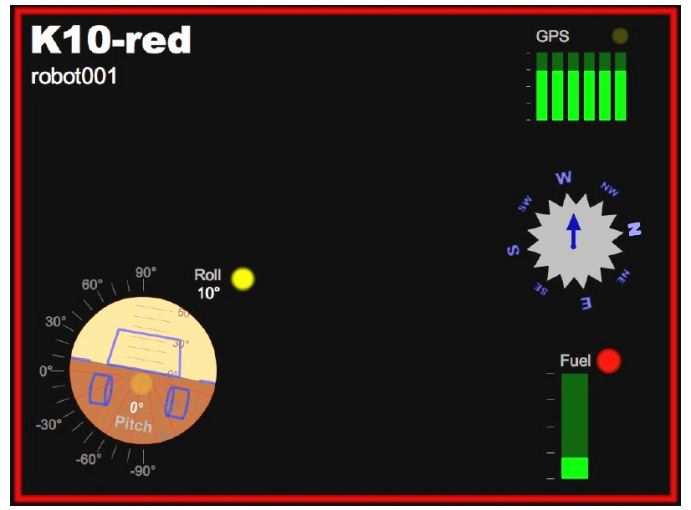

Figure 6. Robot telemonitoring dashboard.

\section{Experimental Results}

We have been using the software and hardware infrastructure described above for the past two months to incrementally test our system and add the features necessary to our overall implementation plan. In this section we describe a selected set of these experiments. 
To establish end-to-end functionality on one prospecting robot we used the K10 at CMU, equipped with an onboard computer responsible for running the Robot Controller and HAD subsystems. Autonomous navigation is achieved with a simple wheel encoder-based method while we complete work on integrating more sophisticated, binocular vision- and GPS-based navigation. HAD currently consists of checking for the robot's heading and attitude as measured by the onboard inertial measurement unit (IMU) and the number of GPS satellelites in view and their signalto-noise ratio; and displaying them on the robot's Dashboard as shown above in Figure 6. The Task Planning and Monitoring tool communicates with the robot via a radio Ethernet socket connection; when the robots being supervised are the SRR at NASA JPL or the K10 at NASA ARC, a secure Internet-based connection is used.

Referring to Figure 4, we defined a set of four waypoints for the robot to traverse (indicated in red), as if a crater or other significant obstacle were present in the field; the last waypoint corresponds to the location where a prospecting activity takes place, e.g., a sample must be obtained. Starting from a position to the left of the upper leftmost waypoint, the robot successfully completed the traverse to the upper rightmost one. From there, the telesupervisor - who was seated in a different room with no direct view of the robot - used the teleoperation station to navigate the robot around the "prospecting site." This is depicted in the photos in Figure 7. During the run, data from the IMU were continuously displayed on the workstation's Dashboard.

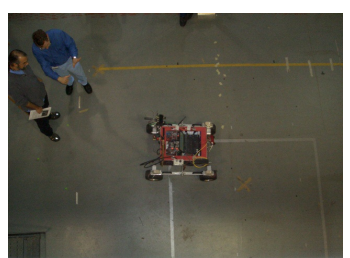

(a)

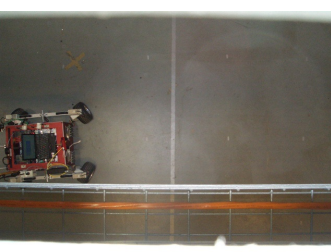

(b)

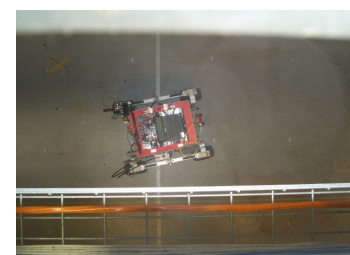

(c)

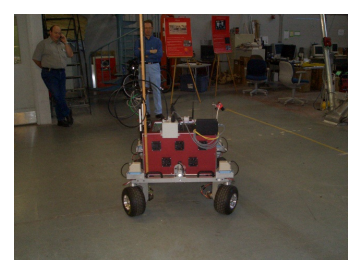

(d)

Figure 7. Autonomous navigation and teleoperation experiment at CMU. (a) Initial position. (b), (c) Traversing through second and third waypoints. (d) "Prospecting" site via teleoperation.

Next, we repeated the teleoperation portion of the experiment with our NASA partners, where the telesupervisor was sitting at the workstation at CMU and the rovers were located at JPL's sandbox and at ARC's Moonscape. Video was transmitted over a somewhat slower Internet connection. Because we don't have, at this moment, stereoscopic cameras installed onboard the SRR or the K10 at NASA ARC, we used simpler web-based cameras, and therefore we used a standard web browser instead of the full StereoGraphics equipment.

Upon completing these experiments, our human telesupervisor reported that the geometrically-correct cameras allowed him to better gauge the distance to the targets. In the future we will perform usability studies of the stereoscopic system to assess operator comfort and efficiency.

Snapshots of these experiments are shown in Figure 8, where we show both the view from the telesupervisor workstation and a local view recorded by a site camera (upper-right inset). 


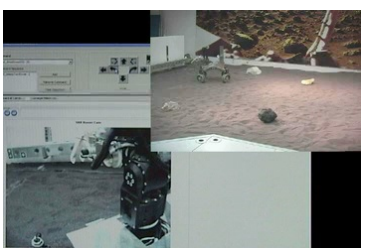

(a)

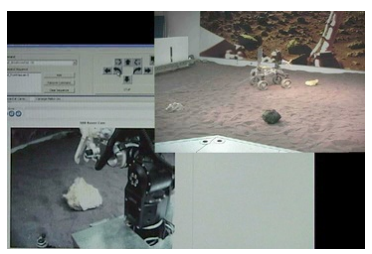

(b)

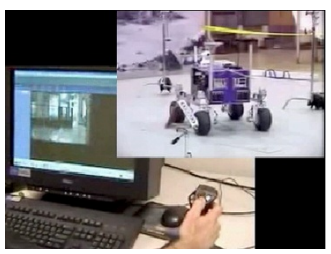

(c)

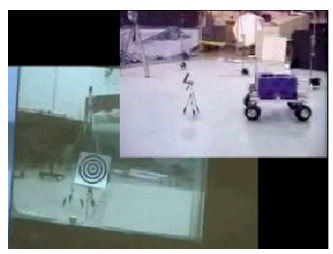

(d)

Figure 8. Teleoperation experiments.

(a) Between CMU and NASA JPL, initial position. (b) At "prospecting" site.

(c) Between CMU and NASA ARC, initial position. (d) At "prospecting" site.

\section{Conclusion}

The work reported by the authors is an ongoing effort to evolve current human-robot interaction technologies to Technology Readiness Level 6 (system/subsystem model or prototype demonstration in a relevant environment - ground or space), and deliver to NASA the means to perform realistic, purposeful, and significant science and exploration once back on the Moon. In the future we will further develop and integrate the subsystems of our architecture, and perform field tests with an increasing number of robots to assess the increase in efficiency and safety it can provide to astronauts.

\section{Acknowledgments}

This work is supported by NASA under Cooperative Agreement No. NNA05CP96A. The authors wish to thank Terrence Fong, Maria Bualat, and Erik Park, from NASA ARC, and Ashitey Trebi-Ollennu, Terry Huntsberger, Ashley Stroupe, Eric Kulczycki, Stephane Smith, Wes Paul, and Diana Acosta, from NASA JPL, for their support during the teleoperation tests described in this paper.

\section{References}

[1] A. Elfes, J.M. Dolan, G. Podnar, S. Mau, M. Bergerman. "Safe and Efficient Robotic Space Exploration with Tele-Supervised Autonomous Robots. AAAI Spring Symposia, Stanford University, USA, March 2006.

[2] A. Elfes. "Incorporating spatial representations at multiple levels of abstraction in a replicated multilayered architecture for robot control." in Intelligent Robots: Sensing, Modelling, and Planning, R. C. Bolles, H. Bunke, H. Noltemeier (eds.), World Scientific, 1997.

[3] T. Fong, C. Thorpe, and C.; Baur. "Multi-robot remote driving with collaborative control." IEEE Transactions on Industrial Electronics, Vol. 50, No. 4, August 2003, pp. 699-704.

[4] F. Heger, L. Hiatt, B.P. Sellner, R. Simmons, and S. Singh, "Results in Sliding Autonomy for Multi-robot Spatial Assembly." 8th International Symposium on Artificial Intelligence, Robotics and Automation in Space, Munich, Germany, September, 2005.

[5] C.D. Kidd and C. Breazeal. "Sociable robot systems for real-world problems." Intl. Workshop on Robot and Human Interactive Communication, Nashville, USA, August 2005, pp. 353-358.

[6] J.C. Mankins. Technology Readiness Levels: A White Paper. NASA Office of Space Access and Technology, April 1995. http://advtech.jsc.nasa.gov/ downloads/ TRLs.pdf.

[7] NASA Mars Exploration Rover Mission. http://marsrovers.jpl.nasa.gov/home/index.html.

[8] I. Nourbakhsh, K. Sycara, M. Koes, et al. "Human-Robot Teaming for Search and Rescue." Pervasive Computing, Vol. 4, No. 1, Jan-Mar 2005, pp. 72-78.

[9] G. Podnar, J. Dolan, A. Elfes, M. Bergerman, H.B. Brown and A.D. Guisewite. "Human Telesupervision of a Fleet of Autonomous Robots for Safe and Efficient Space Exploration." Submitted to Conference on Human-Robot Interaction, Salt Lake City, USA, March 2006.

[10] M. Sierhuis, W.J. Clancey, R.L. Alena, et al. "NASA's Mobile Agents Architecture: A MultiAgent Workflow and Communication System for Planetary Exploration." 8th International Symposium on Artificial Intelligence, Robotics and Automation in Space, Munich, Germany, September 2005. 